\title{
A combined discontinuous Galerkin and finite volume scheme for multi-dimensional VPFP system
}

\author{
M. Asadzadeh, and K. Bartoszek \\ Department of Mathematics, Chalmers University of Technology and University of Gothenburg \\ SE-412 96 Göteborg, Sweden
}

\begin{abstract}
We construct a numerical scheme for the multi-dimensional Vlasov-Poisson-Fokker-Planck system based on a combined finite volume (FV) method for the Poisson equation in spatial domain and the streamline diffusion (SD) and discontinuous Galerkin (DG) finite element in time, phase-space variables for the Vlasov-Fokker-Planck equation.
\end{abstract}

Keywords: Vlasov-Poisson-Fokker-Planck system, finite volume method, streamline diffusion method, discontinuous Galerkin method. PACS: 02.30.Jr, 02.70.Dh, 02.70Hm, 05.20Dd, 47.45.Ab, 51.10.+y.

\section{INTRODUCTION}

We study the approximate solution for the deterministic multi-dimensional Vlasov-Poisson-Fokker-Planck (VPFP) system: given the parameters $\beta$ and $\sigma$ and the initial data $f_{0}(x, v),(x, v) \in \Omega:=\Omega_{x} \times \Omega_{v} \subset \mathbf{R}^{d} \times \mathbf{R}^{d}, d=1,2,3$, find the density function $f(x, v, t)$ in the Dirichlet initial-boundary value problem for the Vlasov-Fokker-Planck equation

$$
\text { (P1) } \begin{cases}f_{t}+v \cdot \nabla_{x} f-\nabla_{x} \varphi \cdot \nabla_{v} f-\operatorname{div}_{v}(\beta v f)-\sigma \Delta_{v} f=S, & \text { in } \Omega \times[0, T], \\ f(x, v, 0)=f_{0}(x, v), & \text { in } \Omega_{x} \times \Omega_{v}, \\ f(x, v, t)=0, & \text { on } \Gamma_{G}^{-} \times[0, T],\end{cases}
$$

where $S$ is source, $G:=\left(v,-\nabla_{x} \varphi\right), \Gamma_{G}^{-}:=\{(x, v) \in \Gamma:=\partial \Omega \mid G \cdot \mathbf{n}<0\}, \mathbf{n}$ is the outward unit normal, and $\varphi$ satisfies

$$
\begin{cases}-\Delta_{x} \varphi=\int_{\Omega_{v}} f(x, v, t) d v, & \text { in } \Omega_{x} \times[0, T], \\ \left|\nabla_{x} \varphi(x, t)\right|=0, \quad(\varphi \text { p.w. constant, or } \varphi=0) & \text { on } \partial \Omega_{x} \times[0, T] .\end{cases}
$$

We solve (P2) replacing $f$ by a given function $g$, and insert the corresponding solution $\varphi_{g}$ in (P1) to obtain an equation for $f$. Thus, we link $f$ to the given data $g$ as, say, $f=\Lambda[g]$. Hence, the solution for the VPFP system is a fixed point of the operator $\Lambda$, i.e. $f=\Lambda[f]$. We may study existence and uniqueness using a Schauder fixed point theorem. For the discrete version this step can be repeated using a Brouwer type fixed point argument, see, e.g. [1] and [3].

Conventional numerical methods for the Vlasov-Poisson and related equations have been dominated by the particle methods see, e.g. [5] and [11]. A 1-dimensional finite volume scheme for the Vlasov-Poisson is studied in [6].

To approximate (P2) we use a finite volume approach in $3 d: \Omega_{x} \subset \mathbf{R}^{3}$. As for (P1) we employ streamline-diffusion and discontinuous Galerkin methods based on the studies in [8] and [4]. We shall only give sketch of the proofs. Detailed proofs are obtained following the techniques in [10] for finite volume, and [1]- [2] and [8] for finite elements.

\section{THE FINITE VOLUME METHOD FOR POISSON EQUATION IN 3D}

The cell-center finite volume (FV) scheme for problem (P2), in standard domain $\Omega_{\mathbf{x}}=(0,1)^{3}$, is given by

$$
-\nabla_{\mathbf{x}}^{2} \varphi=\rho, \quad \text { in } \quad \Omega_{\mathbf{x}}=(0,1) \times(0,1) \times(0,1) \quad\left|\nabla_{x} \varphi\right|=0, \quad \text { on } \quad \partial \Omega_{\mathbf{x}},
$$

where $\rho=\int_{\Omega_{v}} f d v$. Existence uniqueness and regularity studies for (3) are extensions of two-dimensional results in [7]: $\rho \in H^{-1}\left(\Omega_{\mathbf{x}}\right)$ implies that $\exists ! \varphi \in H_{0}^{1}\left(\Omega_{\mathbf{x}}\right)$, and for $\rho \in H^{s}\left(\Omega_{\mathbf{x}}\right)$, with $-1 \leq s<1, s \neq \pm 1 / 2, \varphi \in H^{s+2}\left(\Omega_{\mathbf{x}}\right)$.

Theorem 1. The, respective, optimal FV error estimates for general non-uniform and quasi-uniform meshes are

$$
\left\|\varphi-\varphi_{h}\right\|_{1, h} \leq C h^{s}|\varphi|_{H^{s+1}}, \quad \text { and } \quad\left\|\varphi-\varphi_{h}\right\|_{\infty} \leq C h^{s}|\log h \| \varphi|_{H^{s+1}}, \quad 1 / 2<s \leq 2 ; \quad\left(\text { for }\|\cdot\|_{1, h}\right. \text {, see (7)). }
$$

27th International Symposium on Rarefied Gas Dynamics, 2010

AIP Conf. Proc. 1333, 57-62 (2011); doi: 10.1063/1.3562625

(C) 2011 American Institute of Physics 978-0-7354-0888-0/\$30.00 
The corresponding finite element estimates can be read from the theorem

Theorem 2. a) For the finite element solution of the problem (P2), with a quasiuniform triangulation, we have that:

$$
\left\|\varphi-\varphi_{h}\right\|_{1, \infty} \leq C h^{r}|\log h| \times\|\varphi\|_{r+1, \infty}, \quad r \leq 2
$$

b) $\forall \varepsilon \in(0,1)$ small, $\exists C_{\varepsilon}$ such that $\left\|\varphi-\varphi_{h}\right\|_{1, \infty} \geq C_{\varepsilon} h^{r-\varepsilon}|\log h|$, cf [9].

To derive the finite volume formula we consider the Cartesian mesh

$$
\begin{aligned}
& I_{x}^{h}:=\left\{x_{i}: i=0,1, \ldots, I ; \quad x_{0}=0, \quad x_{i}-x_{i-1}=h_{i} ; \quad x_{I}=1\right\}, \\
& I_{y}^{h}:=\left\{y_{j}: j=0,1, \ldots, J ; \quad y_{0}=0, \quad y_{j}-y_{j-1}=k_{j} ; \quad y_{J}=1\right\}, \\
& I_{z}^{\ell}:=\left\{z_{n}: n=0,1, \ldots, N ; \quad z_{0}=0, \quad z_{n}-z_{n-1}=\ell_{n} ; \quad z_{N}=1\right\} \text {. }
\end{aligned}
$$

With each $\left(x_{i}, y_{j}, z_{n}\right)$ we associate a finite volume box:

$$
\omega_{i j n}=\left(x_{i-1 / 2}, x_{i+1 / 2}\right) \times\left(y_{j-1 / 2}, y_{j+1 / 2}\right) \times\left(z_{n-1 / 2}, z_{n+1 / 2}\right),
$$

and choose central finite volume boxes inside each 27 -points stencil element with the characteristic functions as:

$$
\begin{aligned}
& \left\{\begin{array}{lll}
x_{i-1 / 2}=x_{i}-h_{i} / 2, & x_{i+1 / 2}=x_{i}-h_{(i+1)} / 2, & \hbar_{i}=\frac{h_{i}+h_{i+1}}{2} \\
y_{j-1 / 2}=y_{j}-k_{j} / 2, & y_{j+1 / 2}=y_{j}-k_{(j+1)} / 2, & \bar{k}_{j}=\frac{k_{j}+k_{j+1}}{2} \\
z_{n-1 / 2}=z_{n}-\ell_{n} / 2, & z_{n+1 / 2}=z_{n}-\ell_{(n+1)} / 2, & \bar{\ell}_{n}=\frac{\ell_{n}+\ell_{n+1}}{2}
\end{array}\right. \\
& \left.\chi_{i j n}=\operatorname{Char}\left[\left(-\frac{h_{i+1}}{2}, \frac{h_{i}}{2}\right) \times\left(-\frac{k_{j+1}}{2}, \frac{k_{j}}{2}\right)\right] \times\left(-\frac{\ell_{n+1}}{2}, \frac{\ell_{n}}{2}\right)\right] \in H^{\tau}\left(\mathbf{R}^{3}\right), \quad \forall \tau<1 / 2 .
\end{aligned}
$$

Let now $\rho \in H^{s}\left(\Omega_{\mathbf{x}}\right), s>-1 / 2$, and extend $\rho$ to $\mathbf{R}^{\mathbf{3}}$ preserving its Sobolev class. Thus, we may define

$$
\frac{1}{\left|\omega_{i j n}\right|} \int_{\partial \omega_{i j n}} \frac{\partial \varphi}{\partial \mathbf{n}} d s=\frac{1}{\left|\omega_{i j n}\right|}\left(\chi_{i j n} * \rho\right)\left(x_{i}, y_{j}, z_{n}\right)
$$

using three dimensional convolutions $\chi_{i j n} * \rho$, which is continuous in $\mathbf{R}^{\mathbf{3}}$. Recalling that $\rho \in L_{l o c}^{1}\left(\Omega_{\mathbf{x}}\right)$ we may write

$$
\frac{1}{\left|\omega_{i j n}\right|} \int_{\partial \omega_{i j n}} \frac{\partial \varphi}{\partial \mathbf{n}} d s=\frac{1}{\hbar_{i} \bar{k}_{j} \bar{\ell}_{n}} \int_{x_{i-1 / 2}}^{x_{i+1 / 2}} \int_{y_{j-1 / 2}}^{y_{j+1 / 2}} \int_{z_{n-1 / 2}}^{z_{n+1 / 2}} \rho(x, y, z) d x d y d z
$$

Let $\mathscr{V}_{h}$ be the set of piecewise bilinear functions on the box $\Omega_{\mathbf{x}}$ induced by $\bar{\Omega}_{\mathbf{x}}^{h}$, i.e. $\mathscr{V}_{h}^{\circ}=\left\{F \in \mathscr{V}_{h} \mid F=0 \quad\right.$ on $\left.\quad \partial \Omega_{\mathbf{x}}\right\}$.

Definition 1. The finite volume approximation of the solution $\varphi$ for the Poisson equation: $\varphi_{h} \in \mathscr{V}_{h}^{\circ}$ is defined (implicitly) through the following algorithm:

$$
-\frac{1}{\hbar_{i} \bar{k}_{j} \bar{\ell}_{n}} \int_{\partial \omega_{i j n}} \frac{\partial \varphi_{h}}{\partial \mathbf{n}} d s=\frac{1}{\hbar_{i} \bar{k}_{j} \bar{\ell}_{n}}\left(\chi_{i j n} * \rho\right)\left(x_{i}, y_{j}, z_{n}\right), \quad\left(x_{i}, y_{j}, z_{n}\right) \in \Omega_{\mathbf{x}}^{h} .
$$

Stability and convergence of this method are generalizations of Süli's [10] results in two dimensions for the Dirichlet problem. For $\left|\nabla_{x} \varphi\right|=0$ on $\partial \Omega_{x}$ with extended $\varphi(\infty)=0$ yield $\varphi=0$ on $\partial \Omega_{x}$. The first assertion in Theorem 1 , may be proved repeating the arguments in [10] (we skip) for the $3 \mathrm{~d}$ case in discrete $H^{1}\left(\Omega_{\mathbf{x}}^{h}\right)$ and $L_{2}\left(\Omega_{\mathbf{x}}^{h}\right)$ norms:

$$
\|\psi\|_{1, h}=\left(\|\psi\|^{2}+|\psi|_{1, h}^{2}\right)^{1 / 2}, \quad \text { and } \quad\|\psi\|=(\psi, \psi)^{1 / 2}
$$

where $(\phi, \psi)=\sum_{i=1}^{I-1} \sum_{j=1}^{J-1} \sum_{n=1}^{N-1} \hbar_{i} \bar{k}_{j} \bar{\ell}_{n} \phi_{i j n} \psi_{i j n}$, and $\left.\left.\left.|\psi|_{1, h}=\left(\| \Delta_{x}^{-} \psi \mid\right]_{x}^{2}+\| \Delta_{y}^{-} \psi \mid\right]_{y}^{2}+\| \Delta_{z}^{-} \psi \mid\right]_{z}^{2}\right)^{1 / 2}$, with divided differences $\Delta_{x}^{-} \psi_{i j n}=\left(\psi_{i j n}-\psi_{i-1, j, n}\right) / \hbar_{i}, \Delta_{y}^{-} \psi_{i j n}=\left(\psi_{i j n}-\psi_{i, j-1, n}\right) / \bar{k}_{j}$ and $\Delta_{z}^{-} \psi_{i j n}=\left(\psi_{i j n}-\psi_{i, j, n-1}\right) / \bar{\ell}_{n}$, and the, one-sided discrete $L_{2}$-norms:

$$
\left.\left\|\Delta_{x}^{-} \psi\right\|\right]_{x}^{2}=(\psi, \psi]_{x}, \quad(\phi, \psi]_{x}=\sum_{i=1}^{I} \sum_{j=1}^{J-1} \sum_{n=1}^{N-1} \hbar_{i} \bar{k}_{j} \bar{\ell}_{n} \phi_{i j n} \psi_{i j n}
$$

with similar notations for $y$ and $z$ variables. Our numerical results justify convergence of FV scheme. 


\section{STREAMLINE DIFFUSION AND DISCONTINUOUS GALERKIN APPROACHES}

For a finite element scheme in $\Omega_{T}:=[0, T] \times \Omega$ we use a subdivision of $\Omega$, into the product of tetrahedral elements $\tau_{x}$ and $\tau_{v}$, as $\mathscr{T}_{h}:=\left\{\tau=\tau_{x} \times \tau_{v}\right\}$ combined with a partition of the time interval $(0, T): 0=t_{0}<t_{1}<\ldots<t_{M}=T$, and let $I_{m}:=\left(t_{m}, t_{m+1}\right) ; m=0,1, \ldots, M-1$. Then the corresponding partition of $\Omega_{T}$ is given by the prism-type triangulation

$$
\mathscr{C}_{h}:=\left\{K \mid K:=\tau \times I_{m}, \tau \in \mathscr{T}_{h}, \quad h=\max (\operatorname{diam} \tau)\right\} .
$$

We seek piecewise polynomial approximations for the solution of problem (1) in a finite dimensional space

$$
V_{h}:=\left\{f \in \mathscr{H}:\left.f\right|_{K} \in \mathscr{P}_{k}(\tau) \times \mathscr{P}_{k}\left(I_{m}\right) ; \forall K=\tau \times I_{m} \in \mathscr{C}_{h}\right\},
$$

with $V_{h}$ being continuous in $x$ and $v$, possibly discontinuous in $t$ across time levels $t_{m}$ and $\mathscr{H}:=\prod_{m=0}^{M-1} H^{1}\left(\Omega_{m}\right) ; \Omega_{m}=$ $\Omega \times I_{m}$. We shall also use the jumps $[g]=g^{+}-g^{-}$with $g^{ \pm}=\lim _{s \rightarrow 0 \pm} g(x, v, t+s)$, and the standard notation

$$
\begin{gathered}
(f, g)_{m}=(f, g)_{\Omega_{m}}=\int_{\Omega_{m}} f g d x d v d t, \quad\|g\|_{m}=(g, g)_{m}^{1 / 2}, \quad\langle f, g\rangle_{m}=\int_{\Omega} f\left(\cdot, \cdot, t_{m}\right) g\left(\cdot, \cdot, t_{m}\right) d x d v, \\
|g|_{m}=<g, g>_{m}^{1 / 2}, \quad<f^{\mp}, g^{\mp}>_{\Gamma^{ \pm}}=\int_{\Gamma^{ \pm}} f^{\mp} g^{\mp}\left|G^{h} \cdot n\right| d v, \quad<f^{\mp}, g^{\mp}>_{\lambda \pm}=\int_{I_{m}}<f^{\mp}, g^{\mp}>_{\Gamma^{ \pm}} d t .
\end{gathered}
$$

Using notation $\nabla f:=\left(\nabla_{x} f, \nabla_{v} f\right)$ and $G:=\left(v_{1}, \ldots, v_{d},-\partial \phi / \partial x_{1}, \ldots,-\partial \phi / \partial x_{d}\right)$, we get $\operatorname{div} G(f)=0$. For finite element procedure (both in the SD and the DG cases) we let $\mathscr{F}$ to be a certain (linear) function space, $\tilde{f} \in \mathscr{F}$ an approximation of $f$ and $\Pi f \in \mathscr{F}$ a projection of $f$ into $\mathscr{F}$, then to estimate the approximation error

$$
f-\tilde{f}=(f-\Pi f)+(\Pi f-\tilde{f}) \equiv \eta+\xi ; \quad \xi \in \mathscr{F} .
$$

We use interpolation to estimate $|\|\eta \mid\|$, and establish $|\left\|\xi\left|\|\leq C|| \eta \mid\|, \quad\left(\||| \cdot||:=\| \cdot \|_{\Xi}, \Xi=\mathrm{SD}\right.\right.\right.$ or $\Xi=\mathrm{DG}$, below).

Now we consider the streamline diffusion (SD) method for (P1) with test functions of the form $u+\delta\left(u_{t}+G(\tilde{f}) \cdot \nabla u\right)$ with $\delta \sim h$, the mesh size. For convenience we use the notation $\mathscr{D} w:=w_{t}+G\left(f_{h}\right) \cdot \nabla w$ and formulate the SD method for problem (P1) as follows: given $f_{h}^{-}\left(\cdot, \cdot, t_{m}\right)$, find $f_{h} \in V_{h}$ such that for $m=0, \ldots, M-1$,

$$
\begin{gathered}
\left(P_{m}\right) \quad B_{m}^{\delta}\left(G\left(f_{h}\right) ; f_{h}, u\right)-J_{m}^{\delta}\left(f_{h}, u\right)=L_{m}^{\delta}(u), \quad \forall u \in V_{h} . \\
B_{m}^{\delta}:=\left(\mathscr{D} f_{h}, u+\delta \mathscr{D} u\right)_{m}+\sigma\left(\nabla_{v} f_{h}, \nabla_{v} u\right)_{m}+\left\langle\left[f_{h}\right], u\right\rangle_{m}-\delta \sigma\left(\Delta_{v} f_{h}, \mathscr{D} u\right)_{m}, \\
J_{m}^{\delta}:=\left(\nabla_{v} \cdot\left(\beta v f_{h}\right), u+\delta \mathscr{D} u\right)_{m}, \quad \text { and } \quad L_{m}^{\delta}:=(S, u+\delta \mathscr{D} u)_{m}+\left\langle f^{+}, u^{+}\right\rangle_{\lambda_{m}^{-}}+\left\langle f^{-}, u^{-}\right\rangle_{\lambda_{m}^{+}} .
\end{gathered}
$$

$P_{m}$ is a linear system of equations leading to an implicit scheme and to solve $P_{1}$ is equivalent to find $f_{h} \in V_{h}$ such that

$$
B^{\delta}\left(G\left(f_{h}\right) ; f_{h}, u\right)-J^{\delta}\left(f_{h}, u\right)=L^{\delta}(u), \quad \forall u \in V_{h}, \quad B^{\delta}:=\sum_{m=0}^{M-1} B_{m}^{\delta}, \quad J^{\delta}:=\sum_{m=0}^{M-1} J_{m}^{\delta}, \quad L^{\delta}:=\sum_{m=0}^{M-1} L_{m}^{\delta} .
$$

\section{Stability and error estimates}

Lemma 1. For the $S D$ method we have the coercivity and stability estimates $B^{\delta}\left(G\left(f^{h}\right) ; g, g\right) \geq \frac{1}{2}\|g\|_{S D}^{2}, \forall g \in \mathscr{H}$,

$$
\begin{gathered}
\|g\|_{S D}^{2}=\frac{1}{2}\left[2 \sigma\left\|\nabla_{v} g\right\|_{\Omega_{T}}^{2}+|g|_{M}^{2}+|g|_{0}^{2}+\sum_{m=1}^{M-1}|[g]|_{m}^{2}+2\|\mathscr{D} g\|_{\Omega_{T}}^{2}+\int_{\Gamma \times I} g^{2}\left|G^{h} \cdot n\right|\right], \\
\|g\|_{L_{2}\left(\Omega_{T}, S D\right)}^{2} \leq\left[\frac{1}{C_{1}}\|\mathscr{D} g\|^{2}+\sum_{m=1}^{M-1}|[g]|_{m}^{2}+\int_{\partial \Omega \times I} g^{2}\left|G^{h} \cdot n\right|\right] \delta e^{C_{1} \delta}, \quad \forall C_{1} \geq 0 .
\end{gathered}
$$

Remark 1. In the discontinuous Galerkin case, $\|g\|_{D G}$ and $\|g\|_{L_{2}\left(\Omega_{T}, D G\right)}$ are defined by replacing the $\int$-term, in the $S D$ case, by $\sum \int_{\partial K_{-}\left(G^{\prime \prime}\right)}[g]^{2}\left|G^{h} \cdot n\right| d s$ where $\partial K_{-}\left(G^{\prime \prime}\right)=\left\{(x, v, t) \in \partial K_{-}\left(G^{\prime}\right): n_{t}(x, v, t)=0\right\}$. 
Theorem 3. Assume that there is a constant $C$ such that $\|\nabla f\|_{\infty}+\|G(f)\|_{\infty}+\|\nabla \eta\|_{\infty} \leq C$. Then, we have the following error estimate for the streamline diffusion method for $(P 1)$ :

$$
\left\|f-f_{S D}\right\|_{S D} \leq C h^{k+1 / 2}\|f\|_{H^{k+1}\left(\Omega_{T}\right)} .
$$

Proof. (Sketchy) Let $\tilde{f}^{h}$ be an interpolant of $f$, split the error as $e=f-f_{S D}=f-\tilde{f}^{h}+\tilde{f}^{h}-f_{S D}:=\eta-\xi$. Then,

$$
\begin{array}{r}
\frac{1}{2}\|\xi\|_{S D}^{2} \leq B\left(G\left(f^{h}\right) ; \xi, \xi\right)=B(G(f) ; f, \xi)-B\left(G\left(f^{h}\right) ; \tilde{f}^{h}, \xi\right)+J\left(f^{h}, \xi\right)-J(f, \xi) \\
:=\Delta B+\Delta J \leq \frac{1}{8}\|\xi\|_{S D}^{2}+C_{B}\|\eta\|_{S D}^{2}+\frac{1}{8}\|\xi\|_{S D}^{2}+C_{J}\|\eta\|_{S D}^{2},
\end{array}
$$

where we have used the inverse estimate. The interpolation error $\|\eta\|_{S D}^{2} \leq C_{i} h^{k+1 / 2}\|f\|_{H^{k+1}\left(\Omega_{T}\right)}$, yields the result.

In the DG case, we assume also discontinuities in $x$ and $v$ over the interelement boundaries and use discrete spaces

$$
W_{h}=\left\{g \in L_{2}\left(Q_{T}\right):\left.g\right|_{K} \in P_{k}(K) \quad \forall K \in \mathscr{C}_{h}\right\}, \quad W_{h}^{d}=\left\{w \in\left[L_{2}\left(Q_{T}\right)\right]^{d}:\left.w\right|_{K} \in\left[P_{k}(K)\right]^{d} \quad \forall K \in \mathscr{C}_{h}\right\} .
$$

Theorem 4. Under the assumptions of Theorem 3 and for the exact solution $f \in H^{k+1}\left(\Omega_{T}\right) \cap W^{k+1, \infty}\left(\Omega_{T}\right)$, we have that the discontinuous Galerkin approximation $f_{D G} \in W_{h}^{d}$ for $f$ in $(P 1)$ satisfies the error estimate

$$
\left\|f-f_{D G}\right\|_{D G} \leq C h^{k+1 / 2}\left(\|f\|_{H^{k+1}\left(\Omega_{T}\right)}+\|f\|_{W^{k+1, \infty}\left(\Omega_{T}\right)}\right) .
$$

Proof. (Sketchy) Here we demonstrate only the terms that are involved in estimations of the interelement jump terms, which are additional to those in the SD-case. To this end, we introduce $R: W_{h} \rightarrow W^{d}$, see [4], defined by

$$
R(g) w=-\sum_{\tau_{x} \times I_{m}} \int_{\tau_{x} \times I_{m}} \sum_{e \in E_{v}} \int_{e}[[g]] n_{v} \cdot(w)^{0} d v, \quad \forall w \in W_{h}^{d},
$$

$E_{v}$ is the set of all interior edges of $T_{h}^{v}$. Define $(\chi)^{0}=\frac{\chi+\chi^{e x t}}{2}$ and $[[\chi]]=\chi-\chi^{\text {ext }}$, where $\chi^{\text {ext }}$ is the value of $\chi$ in the element $\tau_{v}^{e x t}$ having $e \in E_{v}$ common edge with $\tau_{v}$. Let $r_{e}$ be the restriction of $R$ to the elements sharing the edge $e \in E_{v}$ :

$$
r_{e}(g) w=-\sum_{\tau_{x} \times I_{m}} \int_{\tau_{x} \times I_{m}} \int_{e}[[g]] n_{v} \cdot(w)^{0} d v, \quad \forall w \in W_{h}^{d} .
$$

Hence, we may easily verify that $\sum_{e \subset \partial \tau_{v} \cap E_{v}} r_{e}=R$ on $\tau_{v} \Longrightarrow\|R(g)\|_{K}^{2} \leq \gamma \sum_{e \subset \partial \tau_{v} \cap E_{v}}\left\|r_{e}(g)\right\|_{K}^{2}$, where $\tau_{v}$ corresponds to the element $K$ and $\gamma=\gamma(d)>0$ is a constant. Furthermore, since the support of each $r_{e}$ is the union of elements sharing the edge $e, \sum_{e \in E v}\left\|r_{e}(g)\right\|^{2}=\sum_{K \in C} \sum_{e \subset \partial \tau_{v} \cap E v}\left\|r_{e}(g)\right\|_{K}^{2}$. The DG method reads as: find $f_{h} \in W_{h}$ such that $B_{D G}\left(G\left(f_{h}\right) ; f_{h}, g\right)-K\left(f_{h}, g\right)=L(g), \forall g \in W_{h},(K f, g)=\left(\nabla_{v}(\beta v f), g+h \mathscr{D} g\right)$. Proving the coercivity: $\left.B_{D G} G\left(f^{h}\right) ; g, g\right) \geq \alpha|\|g\||^{2}, \forall g \in W_{h}$, (compared to $B_{S D}$, contains also interelement jumps) yields the DG estimate.

\section{NUMERICAL EXAMPLES FOR FVM}

Our implementations are done, in $2 \mathrm{~d}$, in the $\mathrm{R}$ statistical package for:

Gaussian function: $\quad u_{1}(x, y)=\exp \left(-\left((\cot (\pi x))^{2}+(\cot (\pi y))^{2}\right)\right) \cdot \chi_{(0,1) \times(0,1)}(x, y), \quad$ with

$$
\Delta u_{1}(x, y)=\frac{1}{2} \pi^{2} \exp \left(-\left((\cot (\pi x))^{2}+(\cot (\pi y))^{2}\right)\right) \cdot\left(\sum_{\tau=x, y} \frac{1+6 \cos (2 \pi \tau)+\cos (4 \pi \tau)}{\sin ^{6}(\pi \tau)}\right) \cdot \chi_{(0,1) \times(0,1)}(x, y) .
$$

Mollifier: $\quad u_{2}(x, y)=\exp \left(-\frac{1}{1-\left(4(y-0.5)^{2}+4(x-0.5)^{2}\right)}\right) \cdot \chi_{(0,1) \times(0,1)}(x, y), \quad$ with

$$
\begin{aligned}
\Delta u_{2}(x, y)= & \exp \left(-\frac{1}{1-\left(4(y-0.5)^{2}+4(x-0.5)^{2}\right)}\right) \cdot\left\{-\frac{128\left((y-0.5)^{2}+(x-0.5)^{2}\right)}{\left(1-\left(4(y-0.5)^{2}+4(x-0.5)^{2}\right)\right)^{3}}+\right. \\
& \left.+\frac{64\left((y-0.5)^{2}+(x-0.5)^{2}\right)}{\left(1-\left(4(y-0.5)^{2}+4(x-0.5)^{2}\right)\right)^{4}}-\frac{16}{\left(1-\left(4(y-0.5)^{2}+4(x-0.5)^{2}\right)\right)^{2}}\right\} \cdot \chi_{(0,1) \times(0,1)}(x, y) .
\end{aligned}
$$



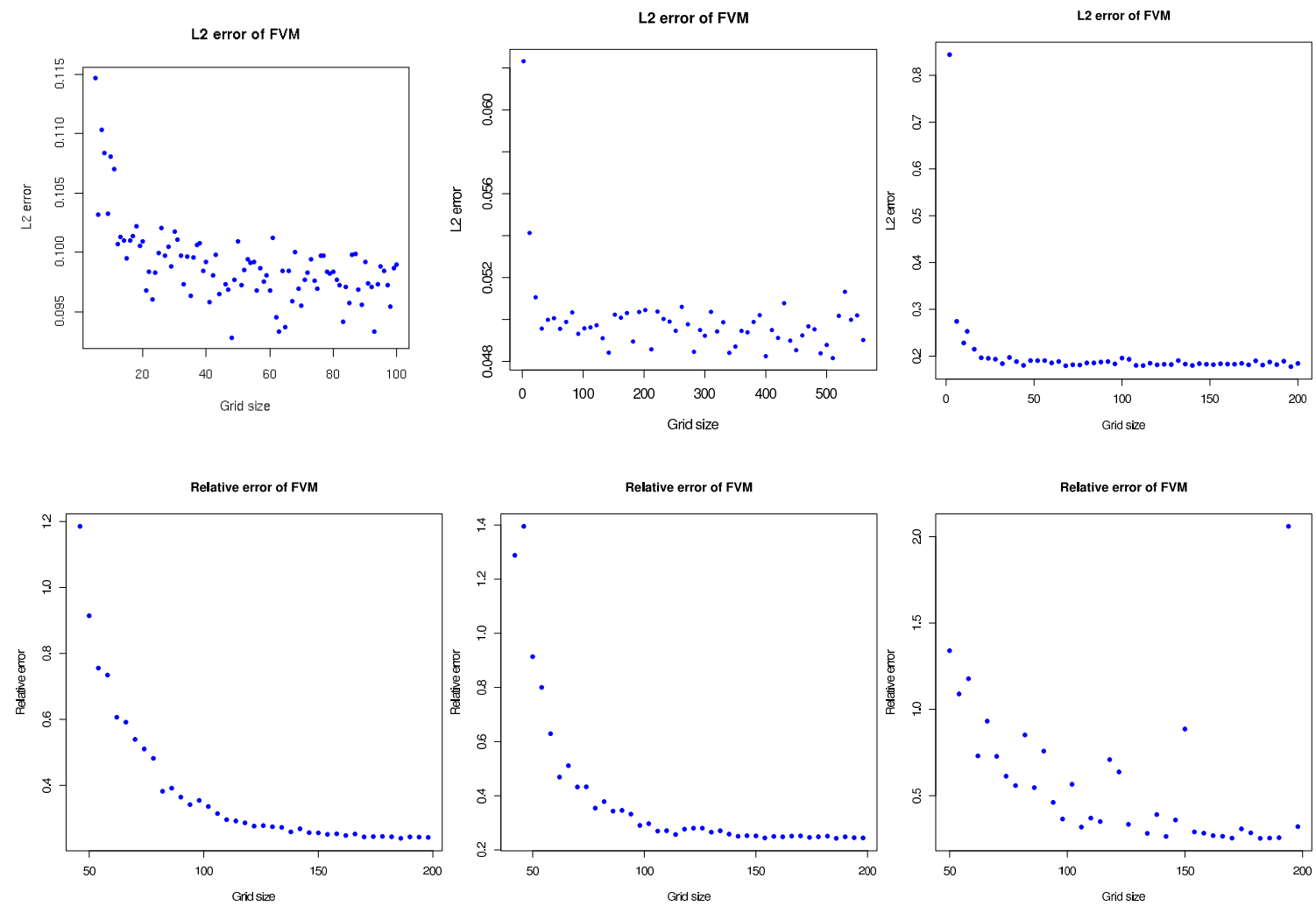

FIGURE 1. Left: error for Gaussian $u_{1}$ function. Center : error for mollifier $u_{2}$ function. Right : potential $u_{3}$ function. Top row: $L^{2}$ errors, bottom row relative errors (the relative errors for small meshes are not shown, as they were extremely high and also the relative error was not evaluated if the value of the true function was less than $10^{-5}$ ).

The FVM performs reasonably well for $u_{1}$ and $u_{2}$. Errors, for different mesh sizes, and graphs are in Figs. 1 and 2.

Mollifier difference - potential: Let $u_{2}(x, y)$ denote the mollifier function defined in section Mollifier. To create a potential we take a difference of two mollifiers, one shrunk to a smaller region.

$$
\begin{aligned}
u_{3}(x, y) & =3 \cdot u_{2}(x, y)-10 \cdot u_{2}\left(\frac{x}{2}-\frac{1}{8}, \frac{y}{2}-\frac{1}{8}\right) \cdot \chi_{\left(\frac{1}{4}, \frac{3}{4}\right) \times\left(\frac{1}{4}, \frac{3}{4}\right)}(x, y) \\
\Delta u_{3}(x, y) & =3 \cdot \Delta u_{2}(x, y)-\frac{10}{4} \cdot \Delta u_{2}\left(\frac{x}{2}-\frac{1}{8}, \frac{y}{2}-\frac{1}{8}\right) \cdot \chi_{\left(\frac{1}{4}, \frac{3}{4}\right) \times\left(\frac{1}{4}, \frac{3}{4}\right)}(x, y)
\end{aligned}
$$

Note that the characteristic function in the definition of $u_{3}$ does not cause any problems for the derivatives as the mollifier goes smoothly to 0 .
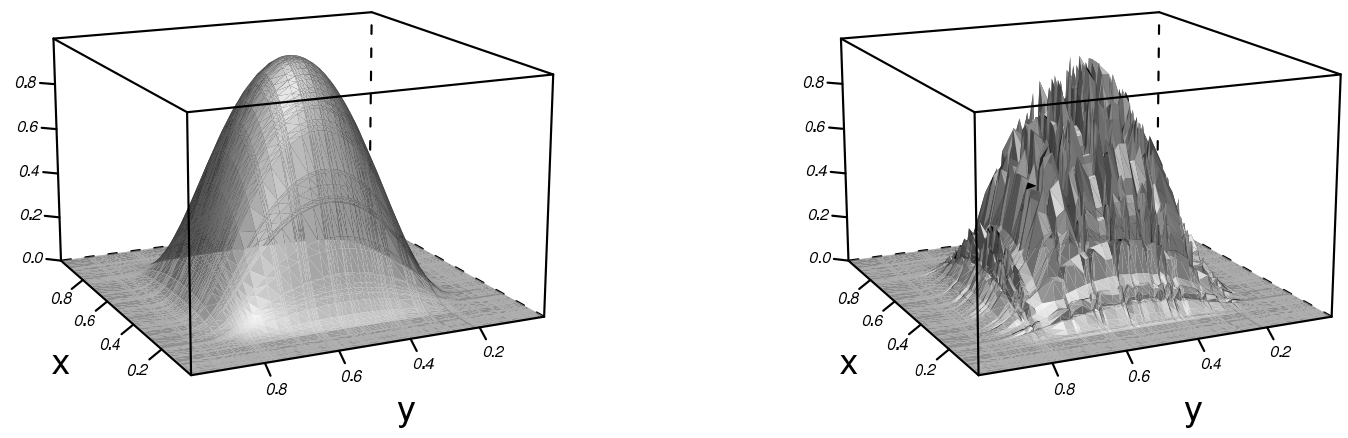

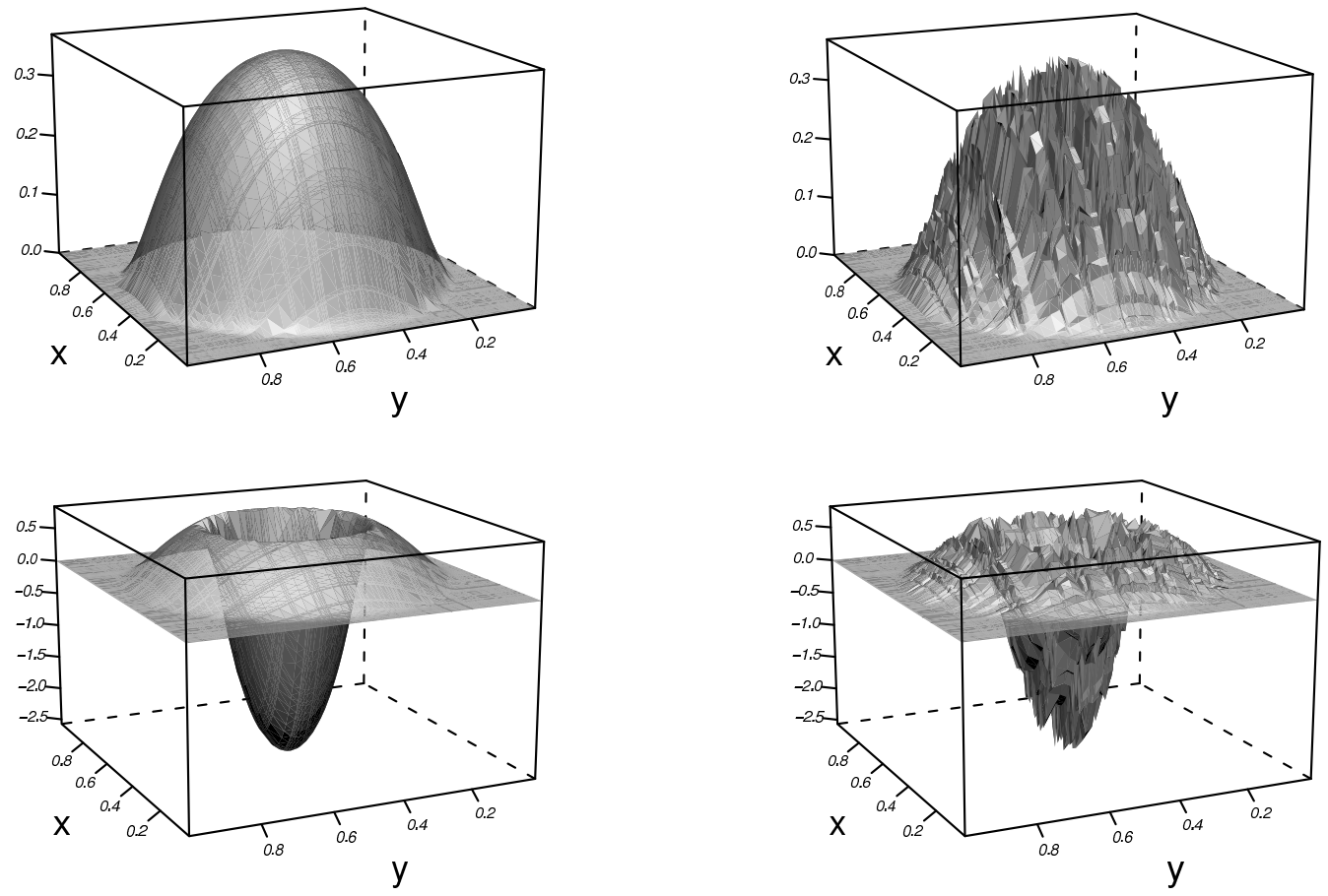

FIGURE 2. Top left : true Gaussian function, top right : FVM approximation of Gaussian function, center left : true mollifier function, center right : FVM approximation of mollifier function, bottom left : true potential function, bottom right : FVM approximation of potential function. The FVM was calculated on a random grid of 50 internal nodes in each dimension. The points were the same in both dimensions. The graphs are done (by wireframe () function) on a random grid (different from the FVM one) of 100 internal nodes in each dimension. The points are the same in both dimensions.

\section{REFERENCES}

1. M. Asadzadeh, Streamline diffusion methods for The Vlasov-Poisson equation, Math. Model. Numer. Anal., 24 (1990), no. 2 , 177-196.

2. M. Asadzadeh and P. Kowalczyk, Convergence of streamline diffusion methods for the Vlasov-Poisson-Fokker-Planck equations, Numer Methods Partial Differential Eqs., 21 (2005), 472-495.

3. F. Bouchut, Smoothing Effect for the Non-linear Vlasov-Poisson-Fokker-PLanck System, J. part. diff. equ., 122 (1995), pp 225-238.

4. F. Brezzi, G. Manzini, D. Marini, P. Pietra and A. Russo, Discontinuous Galerkin approximations for elliptic problems, Numer. Meth. Partial Diff. Equs., 16 (2000), no. 4, 365-378.

5. G. H. Cottet and P. A. Raviart, On particle-in-cell methods for the Vlasov-Poisson equations, Trans. Theory Statist. Phys., 15 (1986), 1-31.

6. F. Filbet, Convergence of a finite volume scheme for the Vlasov-Poisson system, SIAM J. Numer. Anal., 39 (2001), $1146-1169$.

7. P. Grisvard, Elliptic Problems in Non-Smooth Domains, Pitman, 1965.

8. C. Johnson and J. Saranen, Streamline diffusion methods for the incompressible Euler and Navier-Stokes equations, Math. Comp. 47 (1986), 1-18.

9. Y. Lin, V. Thomee and L. Wahlbin, Ritz-Volterra projections to finite-element spaces and applications to integrodifferential and related equations. SIAM J. Numer. Anal. 28 (1991),1047-1070.

10. E. Süli, Convergence of finite volume schemes for Poisson's equation on nonuniform meshes, SIAM, J. Numer. Anal. 26 (1991), no. 5, 14191-1430.

11. S. Wollman, E. Ozizmir and R. Narasimhan, The convergence of the particle method for the Vlasov-Poisson system with equally spaced initial data points, Transport Theory Statist. Phys. 30 (2001), no. 1, 1-62. 